\title{
Dissecting cellulitis of the scalp
}

INSERM

\section{Source}

INSERM. (1999). Orphanet: an online rare disease and orphan drug data base. Dissecting cellulitis of the scalp. ORPHA:345

Dissecting cellulitis of the scalp is a rare chronic suppurative dermatosis of the scalp that mainly affects black men and that is characterized by multiple painful inflammatory follicular and perifollicular nodules, pustules, and abscesses that interconnect via sinus tracts and eventually result in scarring alopecia. 